\title{
Basaloid Squamous Cell Carcinoma of the Head and Neck: Subclassification into Basal, Ductal, and Mixed Subtypes Based on Comparison of Clinico-pathologic Features and Expression of p53, Cyclin D1, Epidermal Growth Factor Receptor, p16, and Human Papillomavirus
}

Kyung-Ja Cho · Se Un Jeong Sung Bae Kim ${ }^{1}$. Sang-wook Lee ${ }^{2}$ Seung-Ho Choi ${ }^{3}$ Soon Yuhl $\mathrm{Nam}^{3}$ Sang Yoon $\mathrm{Kim}^{3}$

Departments of Pathology, ${ }^{1}$ Medical Oncology, ${ }^{2}$ Radiation Oncology, and ${ }^{3}$ Otorhinolaryngology, Asan Medical Center, University of Ulsan College of Medicine, Seoul, Korea

Received: January 17, 2017

Revised: February 21, 2017

Accepted: March 3, 2017

Corresponding Author

Kyung-Ja Cho, MD

Department of Pathology, Asan Medical Center,

University of Ulsan College of Medicine,

88 Olympic-ro 43-gil, Songpa-gu, Seoul 05505,

Korea

Tel: $+82-2-3010-4545$

Fax: +82-2-472-7898

E-mail: kjc@amc.seoul.kr

\begin{abstract}
Background: Basaloid squamous cell carcinoma (BSCC) is a rare variant of squamous cell carcinoma with distinct pathologic characteristics. The histogenesis of BSCC is not fully understood, and the cancer has been suggested to originate from a totipotent primitive cell in the basal cell layer of the surface epithelium or in the proximal duct of secretory glands. Methods: Twenty-six cases of head and neck BSCC from Asan Medical Center, Seoul, Korea, reported during a 14-year-period were subclassified into basal, ductal, and mixed subtypes according to the expression of basal (cytokeratin [CK] 5/6, p63) or ductal markers (CK7, CK8/18). The cases were also subject to immunohistochemical study for CK19, p53, cyclin D1, epidermal growth factor receptor (EGFR), and p16 and to in situ hybridization for human papillomavirus (HPV), and the results were clinico-pathologically compared. Results: Mixed subtype (12 cases) was the most common, and these cases showed hypopharyngeal predilection, older age, and higher expression of CK19, p53, and EGFR than other subtypes. The basal subtype (nine cases) showed frequent comedo-necrosis and high expression of cyclin D1. The ductal subtype (five cases) showed the lowest expression of p53, cyclin D1, and EGFR. A small number of p16- and/or HPV-positive cases were not restricted to one subtype. BSCC was the cause of death in 19 patients, and the average follow-up period for all patients was 79.5 months. Overall survival among the three subtypes was not significantly different. Conclusions: The results of this study suggest a heterogeneous pathogenesis of head and neck BSCC. Each subtype showed variable histology and immunoprofiles, although the clinical implication of heterogeneity was not determined in this study.
\end{abstract}

Key Words: Carcinoma, squamous cell; Keratins; Tumor suppressor protein p53; Receptor, epidermal growth factor; Cyclin D1
Basaloid squamous cell carcinoma (BSCC) of the head and neck is a rare variant of squamous cell carcinoma (SCC) with distinct pathologic characteristics with multipotential differentiation. ${ }^{1}$ Histologic characteristics of BSCC include solid nesting, comedonecrosis, cribriform pattern, trabecular arrangement, ductal differentiation, and associated conventional SCC, most frequently seen in situ. The histogenesis of BSCC is not fully understood, and the cancer has been suggested to originate from a totipotent primitive cell in the basal cell layer of the surface epithelium or in the proximal duct of secretory glands. ${ }^{2}$ The incidence of ductal differentiation in BSCC has been reported to range from $27.5 \%$ $45 \%$ and was associated with significantly better survival than those without ductal differentiation in a study by Imamhasan et $a l .{ }^{4}$
However, the clinical significance of the differentiation profiles of BSCC has not otherwise been studied. Most studies on BSCC cytokeratin $(\mathrm{CK})$ immunoprofiles have focused on diagnostic value and the ability to distinguish BSCC from other carcinomas with basaloid features. ${ }^{5-12}$ CK14 was the most consistently expressed CK in BSCC, ${ }^{3,5,6,10-12}$ which is similar to the pattern seen in conventional SCC. ${ }^{4}$ Nonetheless, considering the histologic diversity of BSCC, CK immunoprofiles of BSCC are expected to be heterogeneous and not unique. The aim of this study was to examine expression patterns of basal and ductal immunomarkers, including variable CKs, in BSCC of the head and neck; to subclassify them according to basal or ductal immunomarker expression; and to compare their histology, other oncogene expression, human pap- 
illomavirus (HPV) status, and clinical features.

\section{MATERIALS AND METHODS}

Immunohistochemistry and in situ hybridization

Twenty-six cases of head and neck BSCC from Asan Medical Center, Seoul, Korea, reported during a 14-year period were subject to immunohistochemical study for CK5/6 (1:200, Zymed, San Francisco, CA, USA), CK7 (1:400, Dako, Glostrup, Denmark), CK8/18 (1:200, Cell Marque, Rocklin, CA, USA), CK19 (1:100, Cell Marque), p63 (1:200, Dako), p53 (1:3,000, Glostrup, cyclin D1 (1:100, Neomarkers, Fremont, CA, USA), epidermal growth factor receptor (EGFR; 1:200, Invitrogen, Carlsbad, CA, USA), p16 ${ }^{\mathrm{INK} 4}$ (1:10, Pharmingen, Franklin Lakes, NJ, USA) and in situ hybridization for HPV. Immunostaining was carried out on 4- $\mu \mathrm{m}$ sections using a Ventana autostainer and an ultraview DAB detection kit (Ventana Medical Systems Inc., Tucson, AZ, USA), according to the manufacturer's instructions. Cytoplasmic staining of CK5/6, CK7, CK8/18, and CK19 and nuclear staining of p63, p53, cyclin D1, and p16 ${ }^{\text {INK4 }}$ were regarded as positive if diffusely or heterogeneously staining was noted in $\geq$ $20 \%$ of the tumor cells. EGFR staining was regarded as positive if $\geq 10 \%$ of tumor cells showed membranous staining with strong or intermediate intensity. For HPV in situ hybridization, the INFORM HPV III Family 16 Probe (B) was used in conjunction with the ISH iView Blue Plus Detection Kit (Ventana Medical Systems Inc.). The INFORM HPV III Family 16 Probe (B) detects the following high-risk HPV genotypes: 16, 18, 31, 33, 35, 39, 45, 51, 52, 56, 58, and 66. Light microscopy results were analyzed, and any blue nuclear dots in the tumor cells were regarded as positive staining.

\section{Clinicopathologic analysis}

Cases that expressed either one or two basal (CK5/6 and p63) or ductal (CK7 and CK8/18) markers were considered as basal or ductal subtypes, and expression of both basal and ductal markers was subclassified into a mixed subtype. The case histologies were re-evaluated for the presence of nuclear palisading, comedonecrosis, cribriform pattern, ductal differentiation, mucin production, and desmoplasia. Clinical information, including survival period of the patients, was obtained via medical records. The average follow-up period for all patients was 79.5 months.

For descriptive statistics, all categorical data were compared with the chi-square test. Continuous data were expressed as mean values and were compared using ANOVA. A survival curve was estimated using the Kaplan-Meier method, and differences in survival between groups were compared using the log-rank test. $\mathrm{p}<.05$ was considered statistically significant.

\section{RESULTS}

\section{Expression of basal and ductal markers}

Positive rates for basal (CK5/6; p63) and ductal markers (CK7; CK8/18) in 26 BSCC are summarized in Table 1. Overall positive rates for $\mathrm{CK} 5 / 6, \mathrm{p} 63, \mathrm{CK} 7$, and $\mathrm{CK} 8 / 18$ were $65.4 \%$, $73.1 \%, 34.6 \%$, and $46.2 \%$, respectively. Twelve combination patterns were observed, and BSCCs were subdivided into basal (nine cases), ductal (five cases), and mixed (12 cases) subtypes (Fig. 1). Clinical features of the 26 cases based on subtype are summarized in Table 2. Advanced age and male gender were more frequently observed in the mixed subtype than in the basal and ductal subtypes. While $60 \%$ of laryngeal or hypopharyngeal BSCC was classified as the mixed subtype, $60 \%$ of oropharyngeal BSCC was determined to be the basal subtype. BSCC was the cause of death in 19 of the 26 patients. Overall survival periods were not significantly different among the three subtypes (Fig. 2).

\section{Histologic parameters}

The relationships between histologic parameters and subtypes are listed in Table 3. Nuclear palisading was observed in both basal and ductal subtypes, but not in the mixed subtype ( $\mathrm{p}=$ .010). Comedo-necrosis was most commonly observed in the basal subtype, followed by ductal and mixed subtypes $(\mathrm{p}=.001)$. Ductal differentiation or mucin production was observed not only in the ductal subtype but also in the basal and mixed subtypes. Desmoplasia was most common in the basal subtype, followed by ductal and mixed subtypes $(\mathrm{p}=.021)$. Representative histo-

Table 1. Combined expression patterns of basal and ductal markers in head and neck basaloid squamous cell carcinomas

\begin{tabular}{lccccc}
\hline & No. of cases & CK5/6 & p63 & CK7 & CK8/18 \\
\hline Basal subtype & 7 & + & + & - & - \\
& 1 & + & - & - & - \\
Ductal subtype & 1 & - & + & - & - \\
& 2 & - & - & + & + \\
Mixed subtype & 2 & - & - & + & - \\
& 1 & - & - & - & + \\
& 1 & + & + & + & + \\
& 5 & + & + & + & - \\
Total, $\mathrm{n}(\%)$ & 1 & + & + & - & + \\
\hline
\end{tabular}

CK, cytokeratin. 

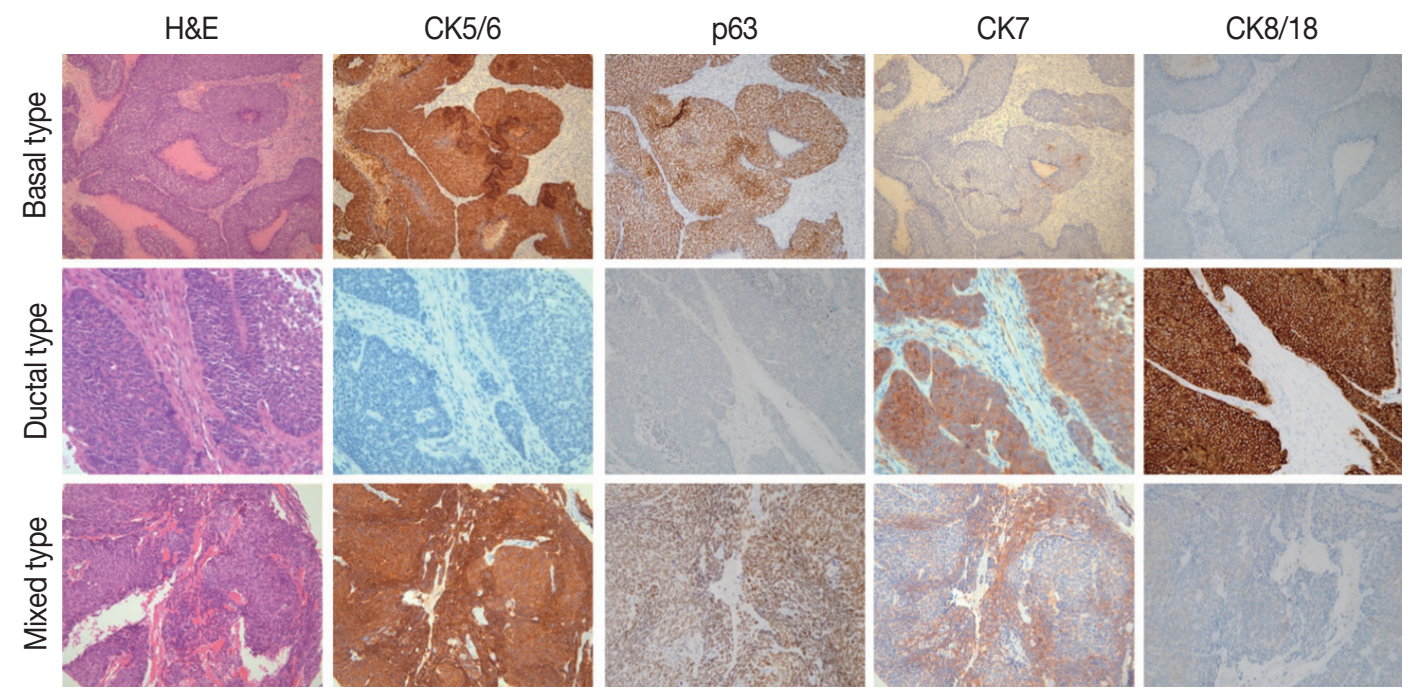

Fig. 1. Examples of combined expression patterns of basal and ductal immunomarkers in head and neck basaloid squamous cell carcinomas. CK, cytokeratin.

Table 2. Clinical features of head and neck basaloid squamous cell carcinoma according to subtype

\begin{tabular}{lccc}
\hline Characteristic & Basal $(n=9)$ & Ductal $(n=5)$ & Mixed $(n=12)$ \\
\hline Age, mean $(y)$ & 59.8 & 58.2 & 63.7 \\
Male:Female & $7: 2$ & $3: 2$ & $11: 1$ \\
Location & & & \\
Larynx $(n=5)$ & 0 & 2 & 3 \\
Hypopharynx $(n=10)$ & 3 & 1 & 6 \\
Oropharynx $(n=5)$ & 3 & 1 & 1 \\
Nasopharynx $(n=1)$ & 1 & - & - \\
Sinonasal $(n=2)$ & 1 & 1 & - \\
Oral cavity $(n=1)$ & - & - & 1 \\
Salivary gland $(n=1)$ & 1 & - & - \\
External auditory canal $(n=1)$ & - & - & 1 \\
Outcome & & & \\
DOD $(n=19)$ & 6 & 4 & 9 \\
AWD $(n=3)$ & 2 & 0 & 1 \\
AUS $(n=4)$ & 1 & 1 & 2 \\
Average survival period (mo) & 65.8 & 59.2 & 52.5 \\
\hline DOD, ded of disease; AWD, alve & &
\end{tabular}

DOD, died of disease; AWD, alive with disease; AUS, alive with unknown status.

logic features are shown in Fig. 3.

\section{Expression of CK19, p53, cyclin D1, EGFR, p16, and HPV}

The expression rates of other tested markers in the three subgroups are compared in Table 4 and illustrated in Fig. 4. CK19, which normally exists in the basal layers of both squamous epithelium and various ductal structures, was positive in $42.3 \%$ of cases and was most commonly identified in the mixed subtype, followed by ductal and basal subtypes ( $\mathrm{p}>.05$ ). Overexpression of p 53 was observed in $34.6 \%$ of cases and was more commonly observed in mixed than in basal or ductal subtypes ( $\mathrm{p}>.05)$. Cyclin

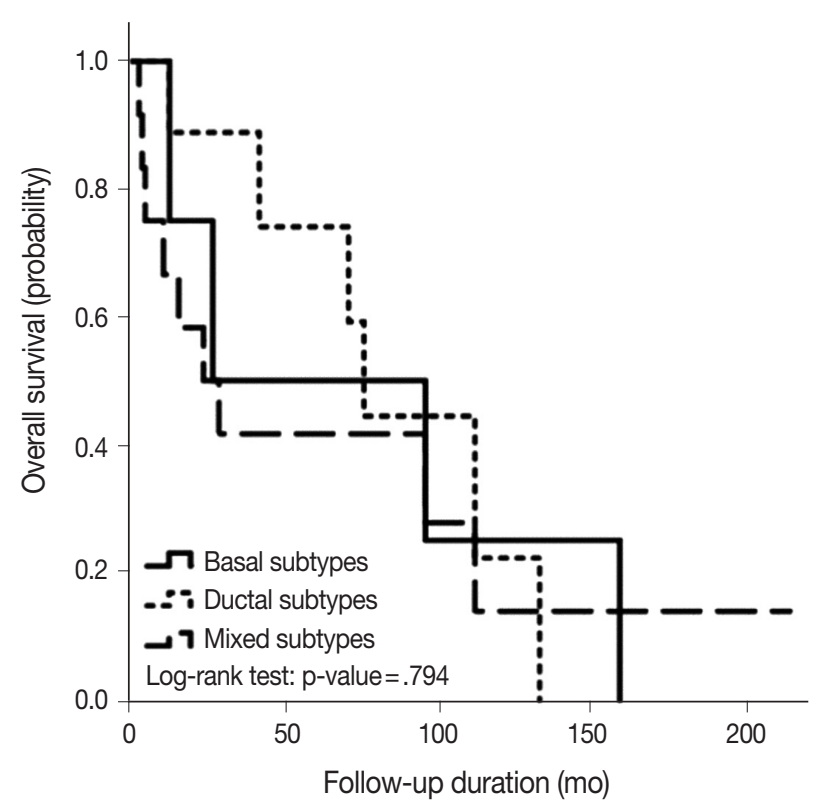

Fig. 2. Overall survival curves of basal, ductal, and mixed subtypes of head and neck basaloid squamous cell carcinomas.

D1 and EGFR expression were observed in $69.2 \%$ and $61.5 \%$, respectively, and the ductal subtype showed the lowest expression rate for both proteins $(\mathrm{p}>$.05). Four p16-positive cases and three $\mathrm{HPV}$-positive cases showed no subtype tendencies.

\section{DISCUSSION}

BSCC is a type of squamous cell carcinoma that occurs in the supraglottic larynx, pyriform sinus, base of tongue, or esophagus. The main histologic components of BSCC are undifferentiated 
cells, but there can be divergent differentiation, including ductal differentiation. The multipotency of BSCC has been described more often with an esophageal origin than in the head and neck. ${ }^{1,3,4}$

Several studies on the CK immunoprofiles of BSCC have been reported, based on diagnoses in the head and neck region, and most of them focused on the diagnostic value and distinguishing BSCC from other carcinomas with basaloid features. ${ }^{5,6,8-12}$ The most frequently expressed CK subtype in BSCC was CK14 (53\%$100 \%)^{5,6,8,10,11}$ and CK5/6 (100\%), ${ }^{9}$ followed by CK19 and CK8/ 18 (80\%), ${ }^{6}$ and CK17 (68\%). ${ }^{5}$ These have been described as useful markers for differentiating BSCC from other histologically similar tumors including basal cell carcinoma with squamous metaplasia, adenoid cystic carcinoma, and neuroendocrine carcinoma. The $\mathrm{CK} 7$ expression rate has varied depending on the report $(0 \%-53 \%)^{8,10,11,13,14}$

Table 3. Histologic features of head and neck basaloid squamous cell carcinoma according to subtype

\begin{tabular}{lcclr}
\hline Histologic finding & $\begin{array}{c}\text { Basal } \\
(\mathrm{n}=9)\end{array}$ & $\begin{array}{c}\text { Ductal } \\
(\mathrm{n}=5)\end{array}$ & $\begin{array}{l}\text { Mixed } \\
(\mathrm{n}=12)\end{array}$ & $\begin{array}{r}\text { Total } \\
(\mathrm{n}=26)\end{array}$ \\
\hline Palisading & $4(44.4)$ & $3(60.0)$ & $0^{\mathrm{a}}$ & $7(26.9)$ \\
Comedonecrosis & $9(100)$ & $3(60.0)$ & $3(25.0)^{\mathrm{b}}$ & $15(57.7)$ \\
Cribriform pattern & $2(22.2)$ & $2(40.0)$ & $3(25.0)$ & $7(26.9)$ \\
Ductal differentiation & $4(44.4)$ & $2(40.0)$ & $2(16.7)$ & $8(30.8)$ \\
Mucin production & $2(22.2)$ & $2(40.0)$ & $3(25.0)$ & $7(26.9)$ \\
Desmoplasia & $4(44.4)$ & $2(40.0)$ & $0^{c}$ & $6(23.1)$ \\
\hline
\end{tabular}

Values are presented as number (\%).

${ }^{\mathrm{a}} \mathrm{p}=.010 ;{ }^{\mathrm{p}} \mathrm{p}=.001 ;{ }^{\mathrm{c}} \mathrm{p}=.021$.
Based on the variable histology and CK expression of BSCC, we hypothesized that this tumor is heterogeneous and not homogenous. Kobayashi et al. ${ }^{3}$ also concentrated on the histologic diversity of esophageal BSCC. They analyzed expression patterns of CK7, CK14, and smooth muscle actin in relationship to different components of BSCCs. However, our attempt to subclassify BSCC according to the expression of basal (CK5/6, p63) or ductal markers (CK7, CK8/18) is novel.

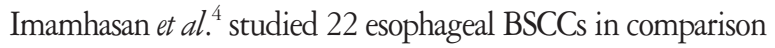
with conventional SCC. BSCC frequently manifested with ductal differentiation (27.5\%), and cases with ductal differentiation were associated with better outcomes than cases without ductal differentiation, although this result was not statistically significant. Ductal differentiation was observed in eight of 26 cases (30.8\%)

Table 4. Other immunomarker expression in head and neck basaloid squamous cell carcinoma according to subtype

\begin{tabular}{llllr}
\hline Immunomarker & $\begin{array}{l}\text { Basal } \\
(\mathrm{n}=9)\end{array}$ & $\begin{array}{c}\text { Ductal } \\
(\mathrm{n}=5)\end{array}$ & $\begin{array}{c}\text { Mixed } \\
(\mathrm{n}=12)\end{array}$ & \multicolumn{1}{c}{$\begin{array}{c}\text { Total } \\
(\mathrm{n}=26)\end{array}$} \\
\hline CK19 & $2(22.2)$ & $2(40.0)$ & $7(58.3)$ & $11(42.3)$ \\
p53 & $2(22.2)$ & $1(20.0)$ & $6(50.0)$ & $9(34.6)$ \\
Cyclin D1 & $7(77.8)$ & $2(40.0)$ & $9(75.0)$ & $18(69.2)$ \\
EGFR & $6(66.7)$ & $1(20.0)$ & $9(75.0)$ & $16(61.5)$ \\
p16 & 1 & 2 & 1 & $4(15.4)$ \\
HPV & 1 & 2 & 0 & $3(11.5)$ \\
\hline
\end{tabular}

Values are presented as number (\%).

CK, cytokeratin; EGFR, epidermal growth factor receptor; HPV, human papillomavirus.
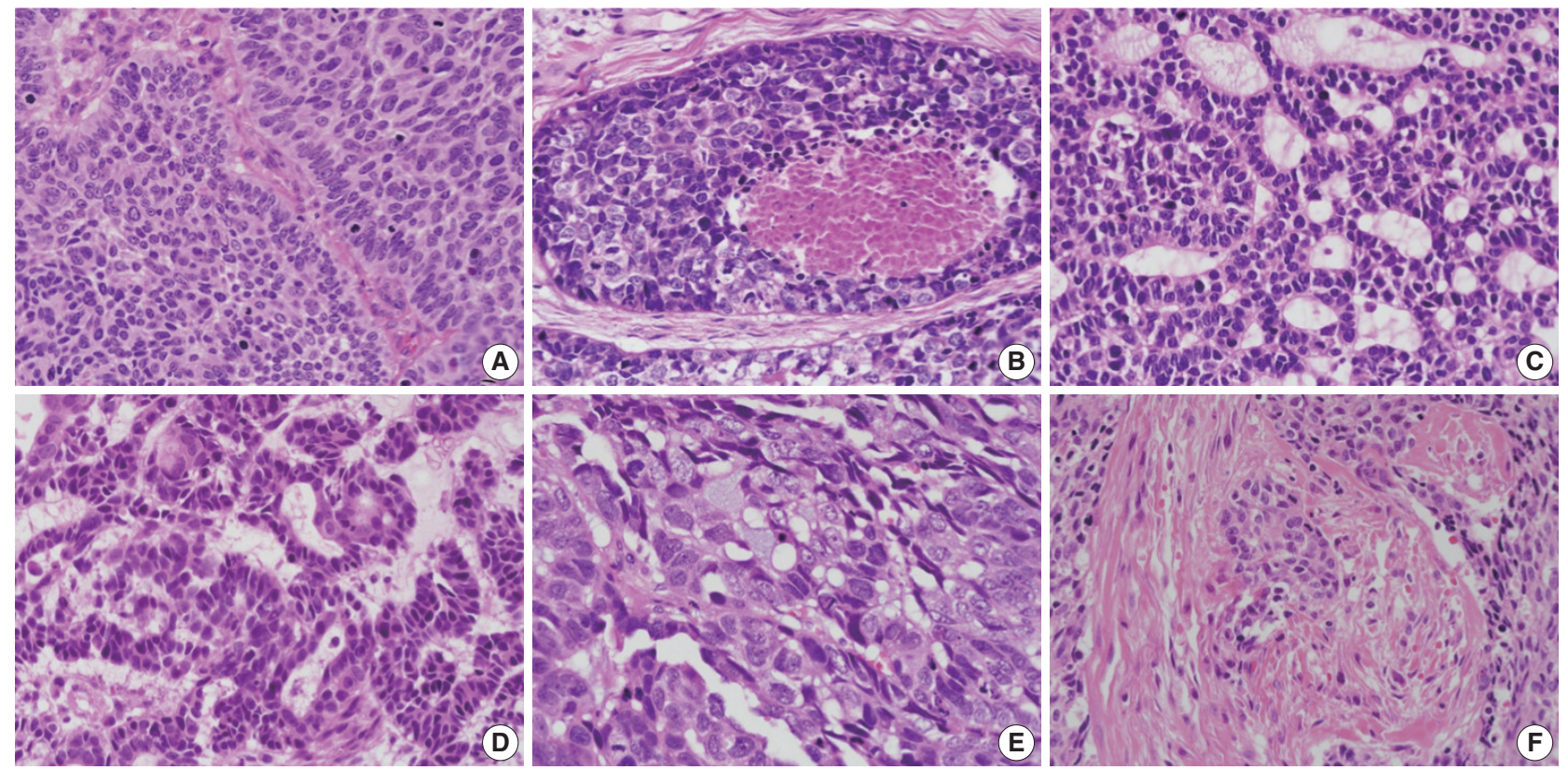

Fig. 3. Representative histologic parameters of basaloid squamous cell carcinomas. (A) Nuclear palisading. (B) Comedo-necrosis. (C) Cribriform pattern. (D) Ductal differentiation. (E) Mucin production. (F) Desmoplasia. 

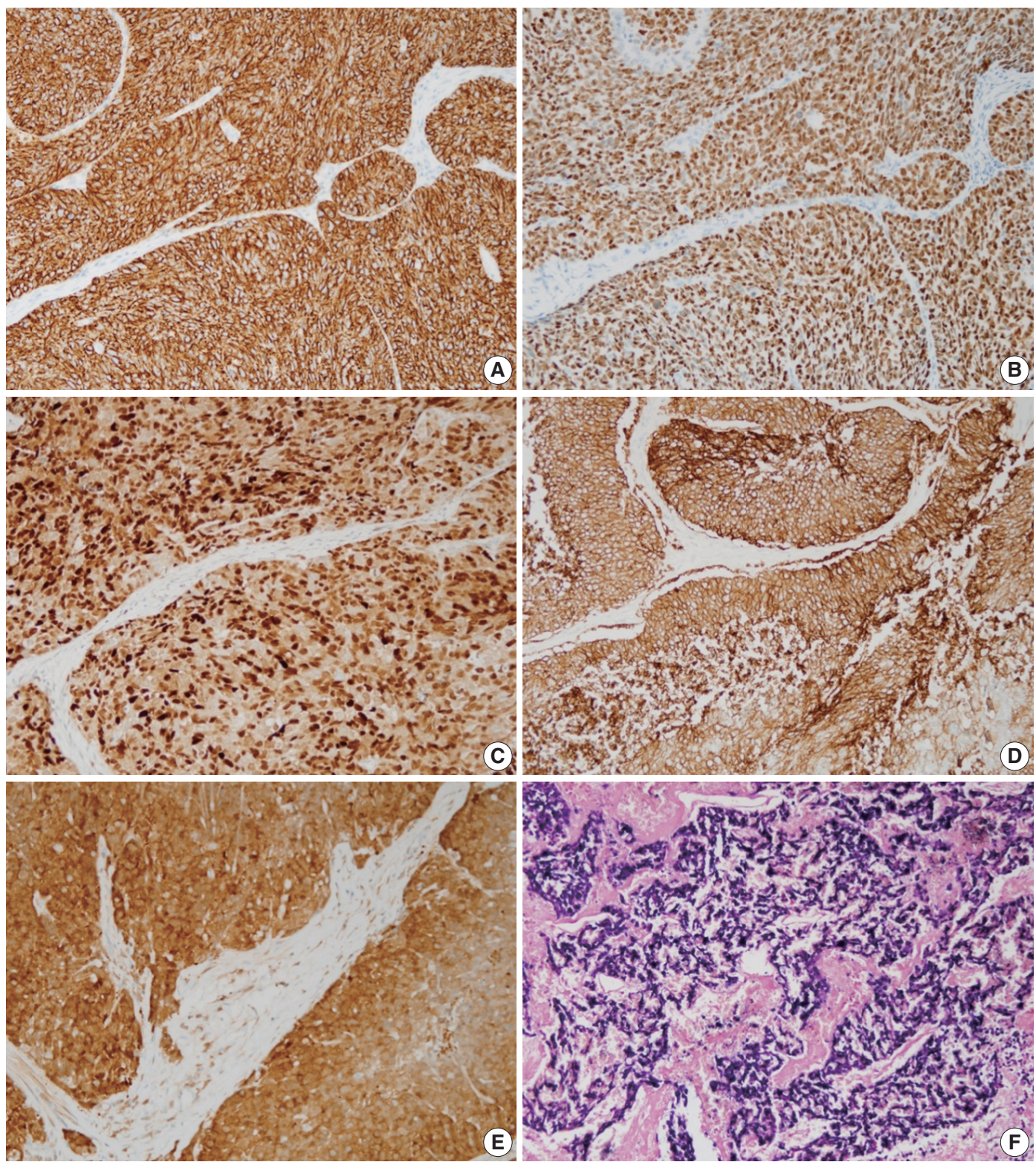

Fig. 4. Representative expression patterns of cytokeratin 19 (A), p53 (B), cyclinD1 (C), epidermal growth factor receptor (D), p16 (E), and human papillomavirus in situ hybridization (F).

in our study. Ductal differentiation or mucin production was not restricted to the ductal subtype but was associated with basal or mixed subtypes. Ductal differentiation and ductal phenotypes were not significantly associated with improved prognoses. There was no significant difference in overall survival among the three subtypes. Among other histologic parameters, peripheral nuclear palisading, comedo-necrosis, and desmoplasia were significantly less frequent in the mixed subtype than in the basal or ductal phenotype. However, the clinical significance of this result was not fully elucidated.

When expression rates for other immunomarkers were com- pared, CK19, which can be both a basal and ductal marker, was positive in $42.3 \%$ of cases, which was lower than the rate of $\mathrm{CK} 5 / 6$ (65.4\%) or CK8/18 (46.2\%) expression. The $\mathrm{p} 53$ expression rate in head and neck BSCC was 34.6\%, which was slightly lower than reported rates of $40 \%-100 \%{ }^{1,6,14,15}$ CyclinD1 and EGFR were positive in $69.2 \%$ and $61.5 \%$, respectively. There are currently no reported data on expression patterns of these proteins in head and neck BSCC. A few studies on esophageal BSCC showed 25\% cyclinD1 positivity and 56\%-100\% EGFR positivity. ${ }^{16,17}$ The ductal subtype tended to exhibit lower levels of $\mathrm{p} 53$, cyclinD1, and EGFR expression than basal or mixed subtypes; however, 
based on the small number of cases in this study, the result was not statistically significant. A small number of p16- and/or HPVpositive cases were not restricted to one subtype. Three HPVpositive cases consisted of one basal subtype from the tonsil, one ductal subtype from the tonsil, and one ductal subtype from the nasal cavity. In the past, a relationship between HPV and basaloid morphology of head and neck squamous cell carcinoma has been suggested, ${ }^{18,19}$ but it is now accepted that not all BSCC cases are associated with HPV, and HPV status is more closely related to the location than histology of SCC. ${ }^{20,21}$ A recent study suggested a relationship between high-risk HPV and CK19 expression, ${ }^{22}$ but two of three HPV-positive cases in this study were negative for CK19.

In summary, BSCC consists of a heterogeneous group that contain tumors with basal, ductal, or mixed immunophenotypes, and this phenomenon suggests a complex histogenesis. Mixed subtypes showed fewer histological characteristics, and the ductal subtype showed less oncogenic protein expression (p53, cyclin D1, and EGFR), although the clinical implications of these findings have not been fully elucidated. The low expression rate of BSCC for CK19 contrasts with the high expression in conventional SCC and limits the diagnostic utility of CK19 mRNA detection in this entity.

Although this study suggested a heterogeneous pathogenesis of head and neck BSCC, there were some limitations to this study, including the retrospective design, small number of cases, and the fact that all patients came from a single institution. Extended or multi-institutional studies might produce more significant results. Furthermore, remarkable advances in investigative tools, such as genomic microarray technologies and next-generation sequencing, could help confirm the results.

\section{Conflicts of Interest}

No potential conflict of interest relevant to this article was reported.

\section{REFERENCES}

1. Cho KJ, Jang JJ, Lee SS, Zo JI. Basaloid squamous carcinoma of the oesophagus: a distinct neoplasm with multipotential differentiation. Histopathology 2000; 36: 331-40.

2. Raslan WF, Barnes L, Krause JR, Contis L, Killeen R, Kapadia SB. Basaloid squamous cell carcinoma of the head and neck: a clinicopathologic and flow cytometric study of 10 new cases with review of the English literature. Am J Otolaryngol 1994; 15: 204-11.
3. Kobayashi $Y$, Nakanishi $Y$, Taniguchi H, et al. Histological diversity in basaloid squamous cell carcinoma of the esophagus. Dis Esophagus 2009; 22: 231-8.

4. Imamhasan A, Mitomi H, Saito T, et al. Immunohistochemical and oncogenetic analyses of the esophageal basaloid squamous cell carcinoma in comparison with conventional squamous cell carcinomas. Hum Pathol 2012; 43: 2012-23.

5. Linskey KR, Gimbel DC, Zukerberg LR, Duncan LM, Sadow PM, Nazarian RM. BerEp4, cytokeratin 14, and cytokeratin 17 immunohistochemical staining aid in differentiation of basaloid squamous cell carcinoma from basal cell carcinoma with squamous metaplasia. Arch Pathol Lab Med 2013; 137: 1591-8.

6. Mane DR, Kale AD, Angadi P, Hallikerimath S. Expression of cytokeratin subtypes: MMP-9, p53, and alphaSMA to differentiate basaloid squamous cell carcinoma from other basaloid tumors of the oral cavity. Appl Immunohistochem Mol Morphol 2013; 21: 431-43.

7. Patil DT, Goldblum JR, Billings SD. Clinicopathological analysis of basal cell carcinoma of the anal region and its distinction from basaloid squamous cell carcinoma. Mod Pathol 2013; 26: 1382-9.

8. Winters R, Naud S, Evans MF, Trotman W, Kasznica P, Elhosseiny A. Ber-EP4, CK1, CK7 and CK14 are useful markers for basaloid squamous carcinoma: a study of 45 cases. Head Neck Pathol 2008; 2: 265-71.

9. Serrano MF, El-Mofty SK, Gnepp DR, Lewis JS Jr. Utility of high molecular weight cytokeratins, but not p63, in the differential diagnosis of neuroendocrine and basaloid carcinomas of the head and neck. Hum Pathol 2008; 39: 591-8.

10. Coletta RD, Almeida OP, Vargas PA. Cytokeratins 1, 7 and 14 immunoexpression are helpful in the diagnosis of basaloid squamous carcinoma. Histopathology 2006; 48: 773-4.

11. Coletta RD, Cotrim P, Almeida OP, Alves VA, Wakamatsu A, Vargas PA. Basaloid squamous carcinoma of oral cavity: a histologic and immunohistochemical study. Oral Oncol 2002; 38: 723-9.

12. Tsubochi H, Suzuki T, Suzuki S, et al. Immunohistochemical study of basaloid squamous cell carcinoma, adenoid cystic and mucoepidermoid carcinoma in the upper aerodigestive tract. Anticancer Res 2000; 20: 1205-11.

13. Xie S, Bredell M, Yang H, Shen S, Yang H. Basaloid squamous cell carcinoma of the maxillary gingiva: a case report and review of the literature. Oncol Lett 2014; 8: 1287-90.

14. Yu GY, Gao Y, Peng X, Chen Y, Zhao FY, Wu MJ. A clinicopathologic study on basaloid squamous cell carcinoma in the oral and maxillofacial region. Int J Oral Maxillofac Surg 2008; 37: 1003-8.

15. Sampaio-Góes FC, Oliveira DT, Dorta RG, et al. Expression of PCNA, p53, Bax, and Bcl-X in oral poorly differentiated and basaloid squamous cell carcinoma: relationships with prognosis. Head Neck 
2005; 27: 982-9.

16. Sato-Kuwabara Y, Fregnani JH, Jampietro J, et al. Comparative analysis of basaloid and conventional squamous cell carcinomas of the esophagus: prognostic relevance of clinicopathological features and protein expression. Tumour Biol 2016; 37: 6691-9.

17. Abe K, Sasano H, Itakura Y, Nishihira T, Mori S, Nagura H. Basaloidsquamous carcinoma of the esophagus: a clinicopathologic, DNA ploidy, and immunohistochemical study of seven cases. Am J Surg Pathol 1996; 20: 453-61.

18. Mendelsohn AH, Lai CK, Shintaku IP, et al. Histopathologic findings of HPV and p16 positive HNSCC. Laryngoscope 2010; 120: 1788-94.

19. El-Mofty SK, Patil S. Human papillomavirus (HPV)-related oropharyngeal nonkeratinizing squamous cell carcinoma: characterization of a distinct phenotype. Oral Surg Oral Med Oral Pathol
Oral Radiol Endod 2006; 101: 339-45.

20. Begum S, Westra WH. Basaloid squamous cell carcinoma of the head and neck is a mixed variant that can be further resolved by HPV status. Am J Surg Pathol 2008; 32: 1044-50.

21. Chernock RD, Lewis JS Jr, Zhang Q, El-Mofty SK. Human papillomavirus-positive basaloid squamous cell carcinomas of the upper aerodigestive tract: a distinct clinicopathologic and molecular subtype of basaloid squamous cell carcinoma. Hum Pathol 2010; 41: 1016-23.

22. Santoro A, Pannone G, Ninivaggi R, et al. Relationship between CK19 expression, deregulation of normal keratinocyte differentiation pattern and high risk-human papilloma virus infection in oral and oropharyngeal squamous cell carcinoma. Infect Agent Cancer 2015; 10: 46. 